\title{
Pityriasis Rubra Pilar with Preceeding Herpes Infection in Childhood - Successful Treatment with Methotrexate
}

\section{Priscilla Filippo Alvim de Minas Santos*}

Hospital Municipal Jesus. Rio de Janeiro, RJ, Brazil

*Corresponding Author: Priscilla Filippo Alvim de Minas Santos, Hospital

Municipal Jesus. Rio de Janeiro, RJ, Brazil.
Received: March 16, 2020

Published: May 04, 2020

(C) All rights are reserved by Priscilla Filippo

Alvim de Minas Santos.

\begin{abstract}
The Pityriasis rubra pilaris (PRP) is a rare, inflammatory, chronic disease of unknown etiology characterized by follicular papules, pinkish-yellow squamous plaques and palmoplantar keratoderma. The diagnosis clinical is most important and confirmed by biopsy of the skin. Many times, the same other clinical aspect could be confused such as psoriasis and atopic dermatitis. Currently, the first-line therapy is the systemic retinoid and other treatments, like methotrexate. The association with viral disease is uncommon. Our purpose was to report a case of PRP in a young boy associated with preceeding with primoinfection by herpes simplex with a favorable response to oral treatment with methotrexate and without recurrence after 10 months of follow-up.
\end{abstract}

Keywords: Methotrexate; Pityriasis Rubra Pilaris; Herpes Infection

\section{Introduction}

Pityriasis rubra pilaris (PRP) is a rare, inflammatory, chronic disease of unknown etiology. The clinical appearance of PRP is highly variable, as is the individual prognosis. The most common presentation is characterized by follicular papules, pinkish-yellow squamous plaques and palmoplantar keratoderma. There are areas of unaffected skin our "islands of sparing" [1,2]. The most patients develop pityriasis rubra pilaris are over 50 years of age, but in individuals of any age, race and nationality may be affected [3]. The estimated incidence is 1 in 400.000 and the precise prevalence is unknown [4]. There are reports in the literature of association with immune system dysregulation, abnormal metabolism of vitamin A, autoimmune disease, infection and malignancy. But it is not common association with primoinfection by herpes simplex [1].

Griffiths proposed its classification in five groups based on clinical appearance, behavior and prognosis [5]. A sixth type was proposed, associated with HIV [6].

The diagnosis of pityriasis rubra pilaris clinic, but can be challenging to make, because it can be confused with psoriasis and atopic dermatitis. The skin biopsy confirms the diagnosis [4].

Currently, the first-line therapy is the systemic retinoid and other treatments, like methotrexate can be possible [7].

\section{Case Report}

H.S, male, 1 year and 8 months, from Rio de Janeiro/Brazil, presented intermittent fever and upper respiratory tract infections. After the 15 days, the boy had onset the lesions of erythema and desquamation on the scalp bilaterally, with follow up face, axial, trunk and limbs, the aspect symmetrical and diffuse psoriform plaques of rosy-orange coloration, associated with islets of normal skin. Presence of lesion the region palmoplantar with hyperkeratosis (Figure 1a-1d).

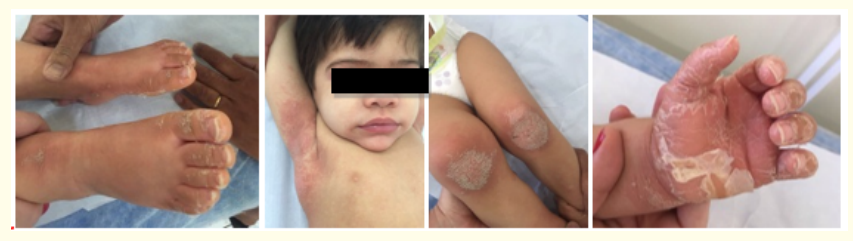

Figure 1a-1d: Clinical presentation of the patient before the treatment with metotrexate.

The emergency pediatrician made the diagnosis of atopic dermatitis and referred to the allergist and immunologist. The personal and family history for atopic diseases and psoriasis was negative. The diagnosis of atopic dermatitis was discarded by them and the dermatologist was performed the clinical diagnosis of pityriasis rubra pilaris.

Complementary tests were performed and showed leukocytosis and serology for herpes simplex virus I and II reactive IgM and non-reactive IgG.

The serology for HIV I and II, HTLV II and II, Parvovirus B19, Epstein Barr, CMV, coxsackie A and B, non-reactive IgM and IgG. FAN non-reactive. The urinoculture and EPF negative. 
The skin biopsy was performed, showing follicular infundibula filled by parakeratotic corneous laminae and the epidermis alternating areas of parakeratosis and orthokeratosis, with discrete perivascular mononuclear inflammatory infiltration in the superficial dermis (Figure 2a and 2b).
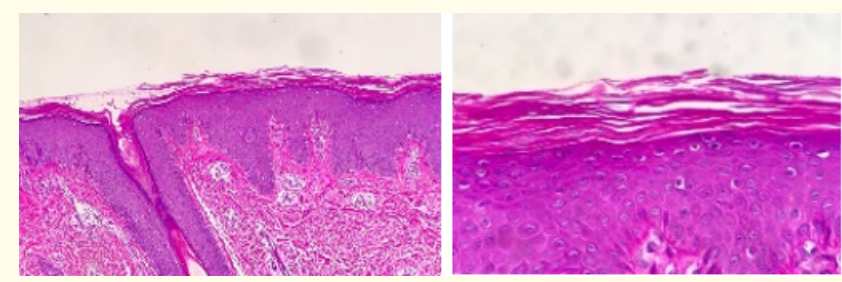

Figure 2a-2b: Histopatology of a biopsy taken form a skin lesion of the right leg (hematoxylin and eosin, original magnification $\mathrm{x} 100$ ).

The treatment initiated was oral with methotrexate $2.5 \mathrm{mg} /$ week for 7 weeks. The patient presented with remission of the clinical picture. The medication was suspended by the child's mother, on his own, because she doesn't "wanted the continuous the treatment". Despite abrupt withdrawal of the medication, the lesions did not return. After 8 months of discontinuation of treatment, the follow up did not show recurrence (Figure 3a-3d).
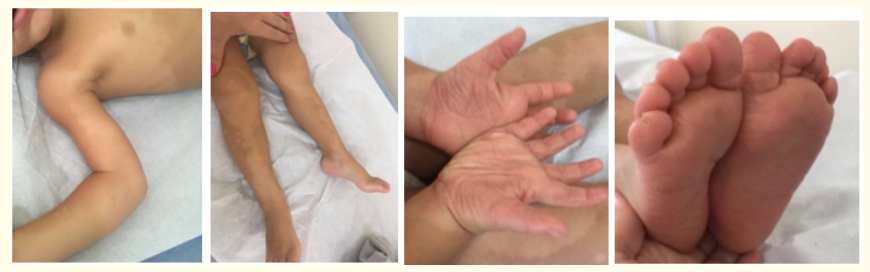

Figure 3a-3d: Clinical presentation of the paciente after 4 moths of oral methotrexate.

\section{Discussion}

PRP is rare pathology in childhood, specially in the age under two years. There are two peaks of onset: one during the first decade of life and a second one in the sixth and seventh decade of life [4]. The disease equally occurs in both sexes [8].

The diagnosis is essentially clinical and confirmed by biopsy. The histopathology reveled a follicular infundibula filled by parakeratotic corneous laminae and the epidermis alternating areas of parakeratosis and orthokeratosis, referred to as the "checkerboard pattern", as in the literature [4].

Most times the lesions are confused with other dermatological diseases such as atopic dermatitis and psoriasis. In the case reported, the diagnosis was initially made of atopic dermatitis and subsequently of pityriasis rubra pilaris. The histopathology confirmed the diagnostic. Acantholysis and focal acantholytic dyskera- tosis within the epidermis have been described in the literature, features which have been suggested to be helpful in distinguishing PRP from psoriasis [4].

Most cases of PRP are acquired and only few reports have been described as familial variants, including autosomal dominant as well as autosomal recessive forms [8].

The etiology is unknown, but there is a report of association with myasthenia gravis, sprue, hypothyroidism, trauma, neoplasias, infectious diseases (HIV, cytomegalovirus infection, chronic hepatitis $\mathrm{C}$ ), but rare herpes simplex primoinfection $[1,8]$. The patient had high respiratory symptoms and fever two weeks before the appearance of the lesions and in the complementary exams the reactive IgM and non-reactive IgG for Herpes simplex, confirming the infectious etiology.

The patient in this case report presented with remission of the clinical picture after 7 weeks of treatment. Ross NA., et al. reported that only $28 \%$ of adult and pediatric patients had entered remission before their study was completed, suggesting that PFP, can be a self-limiting condition in some cases and can persist for $2-3$ years in others cases [4].

Most reports on therapy for PRP are based on empirical data because of the lack of large-scale randomized trials [8]. The retinoids are the most described treatments in both children and adult [9], but a few cases have been reported as a successful therapy using methotrexate in children [10]. In patients who fail to respond to systemic retinoids or in whom the use of retinoid therapy is contraindicated, methotrexate was proposed as a second-line systemic therapy [2]. A cohort of 100 patients indicated that the most commonly used systemic therapies were retinoids (64\%), methotrexate (42\%) and light therapy (26\%) [4]. Griffiths WAD reported a response rate to methotrexate of only 39\%. Our option of treatment was the use of the methotrexate was prescribed, for seven weeks.

Although, the literature does not agree, with the abrupt cessation of methotrexate medication, because it may cause rebound. Our patient did not presented new lesions and after 14 weeks.

The patient had complete remission of the lesions, except with residual discrete hypochromia. After 10 months of follow-up, there was no recurrence of the condition.

\section{Conclusion}

This is a case rare showed the association of the PRP and primoinfection for herpes. The option a favorable oral treatment response with methotrexate for pityriasis rubra pilaris in children, was well tolerated by the patient and can be considered as a good option in this case, with success of treatment. 


\section{Bibliography}

1. Polcari IC and Boyod AH. "Methotrexate treatment in a case of juvenile pityriasis rubra pilaris". Pediatric Dermatology 35.1 (2017): e62-e63.

2. Roenneberg S and Biedermann T. "Pityriasis rubra pilaris: algorithms for diagnosis and treatment". Journal of the European Academy of Dermatology and Venereology 32.6 (2018): 889898.

3. Allison DS., et al. "Pityriasis rubra pilaris in children". Journal of the American Academy of Dermatology 47.3 (2002): 386389.

4. Ross NA., et al. "Epidemiologic, Clinicopathologic, Diagnostic, and Management Challenges of Pityriasis Rubra Pilaris: A Case Series of 100 Patients". JAMA Dermatology 152.6 (2016): 670675.

5. Griffiths WAD. "Pityriasis rubra pilaris". Clinical and Experimental Dermatology 5.1 (1980): 105-112.

6. Miralles ES., et al. "Pityriasis rubra pilaris and human immunodeficiency virus infection". British Journal of Dermatology 133.6 (1995): 990-993.

7. Franzotti AM., et al. "Pitiríase Rubra Pilar e hipotireoidismo". Anais Brasileiros de Dermatologia 89.3 (2014): 497-500.

8. Ivanova K., et al. "Pityriasis rubra pilaris: treatment with biologics - a new promising therapy?" Dermatology 224.2 (2012): 120-125.

9. Alazemi A., et al. "Juvenile pityriasis rubra pilaris: successful treatment with methotrexate". Clinical and Experimental Dermatology 43.1 (2017): 110-112.

10. Genmmeke A., et al. "Pityriasis rubra pilaris - a retrospective single center analysis over eight years". Journal der Deutschen Dermatologischen Gesellschaft 8.6 (2010): 439-444.

\section{Assets from publication with us}

- Prompt Acknowledgement after receiving the article

- Thorough Double blinded peer review

- Rapid Publication

- Issue of Publication Certificate

- High visibility of your Published work

Website: www.actascientific.com/

Submit Article: www.actascientific.com/submission.php Email us: editor@actascientific.com

Contact us: +919182824667 